\title{
PARTITIONS, IRREDUCIBLE CHARACTERS, AND INEQUALITIES FOR GENERALIZED MATRIX FUNCTIONS
}

\author{
THOMAS H. PATE
}

\begin{abstract}
Given a partition $\alpha=\left\{\alpha_{1}, \alpha_{2}, \ldots, \alpha_{s}\right\}, \alpha_{1} \geq \alpha_{2} \geq \cdots \geq \alpha_{s}$, of $n$ we let $X_{\alpha}$ denote the derived irreducible character of $S_{n}$, and we associate with $\alpha$ a derived partition

$$
\alpha^{\prime}=\left\{\alpha_{1}-1, \alpha_{2}-1, \ldots, \alpha_{t}-1, \alpha_{t+1}, \ldots, \alpha_{s}, 1^{t}\right\}
$$

where $t$ denotes the smallest positive integer such that $\alpha_{t}>\alpha_{t+1} \quad\left(\alpha_{s+1}=\right.$ $0)$. We show that if $Y$ is a decomposable $\mathbb{C}$-valued $n$-linear function on $\mathbb{C}^{m} \times \mathbb{C}^{m} \times \cdots \times \mathbb{C}^{m} \quad(n$-copies $)$ then $\left\langle X_{\alpha} Y, Y\right\rangle \geq\left\langle X_{\alpha^{\prime}} Y, Y\right\rangle$. Translating into the notation of matrix theory we obtain an inequality involving the generalized matrix functions $d_{X_{\alpha}}$ and $d_{X_{\alpha^{\prime}}}$, namely that

$$
\left(X_{\alpha}(e)\right)^{-1} d_{X_{\alpha}}(B) \geq\left(X_{\alpha^{\prime}}(e)\right)^{-1} d_{X_{\alpha^{\prime}}}(B)
$$

for each $n \times n$ positive semidefinite Hermitian matrix $B$. This result generalizes a classical result of I. Schur and includes many other known inequalities as special cases.
\end{abstract}

\section{INTRODUCTION}

If $c \in \mathbb{C} S_{n}$, the group algebra obtained from $\mathbb{C}$ and the symmetric group on $\{1,2, \ldots, n\}$, then we define the generalized matrix function $d_{c}$ by

$$
d_{c}(B)=\sum_{\sigma \in S_{n}} c(\sigma) \prod_{i=1}^{n} b_{i \sigma(i)}
$$

for each $n \times n$ matrix $B=\left[b_{i j}\right]$. If $c(e) \neq 0$ then by $\bar{d}_{c}$ we mean $(c(e))^{-1} d_{c}$. Of particular interest are the immanents, the generalized matrix functions $d_{X}$ where $X$ is an irreducible character of $S_{n}$. Familiar examples are $\operatorname{det}(\cdot)$, the determinant function, obtained by setting $c=\varepsilon$, the signum function, and $\operatorname{per}(\cdot)$, the permanent function, obtained by setting $c(\sigma)=1$ for each $\sigma \in S_{n}$.

There are many known inequalities that involve restricting the functions $d_{c}$ to $\mathscr{H}_{n}$, the $n \times n$ positive semidefinite Hermitian matrices. Perhaps the oldest

\footnotetext{
Received by the editors May 31, 1989.

1980 Mathematics Subject Classification (1985 Revision). Primary 15A15; Secondary 15A69,

Key words and phrases. Generalized matrix function, tensor product, induced character, part-
} $15 \mathrm{~A} 45$. ition. 
is the classical Fischer inequality, which states that if $B \in \mathscr{H}_{n+p}$ is partitioned in the form

$$
\left[\begin{array}{ll}
B_{11} & B_{12} \\
B_{21} & B_{22}
\end{array}\right]
$$

where $B_{11}$ is $n \times n$ and $B_{22}$ is $p \times p$ then

$$
\operatorname{det}(B) \leq \operatorname{det}\left(B_{11}\right) \operatorname{det}\left(B_{22}\right)
$$

with equality if and only if $B$ has a row of zeroes or $B_{12}$ is the zero matrix.

In 1908 Schur, see [3], proved that if $X$ is a character of $S_{n}$ then

$$
\operatorname{det}(B) \leq \bar{d}_{X}(B)
$$

for each $B \in \mathscr{H}_{n}$. For a short proof see [4]. Hence, the determinant function is, in the sense of (1.3), the smallest of the normalized immanents. This naturally led to speculation as to which, if any, of the normalized immanents might be largest, in the sense of (1.3).

In the sixties M. Marcus proved, see [5], a partial analogue to (1.2) involving permanents, namely that if $B=\left[b_{i j}\right] \in \mathscr{H}_{n}$ then

$$
\operatorname{per}(B) \geq b_{11} \operatorname{per}(B(1 \mid 1))
$$

where $B(s \mid t), 1 \leq s, t \leq n$, denotes the $(n-1) \times(n-1)$ matrix obtained from $B$ by deleting the sth row and $t$ th column.

Moreover, Marcus conjectured that if $B \in \mathscr{H}_{n+p}$ is partitioned as in our statement of the Fischer inequality then

$$
\operatorname{per}(B) \geq \operatorname{per}\left(B_{11}\right) \operatorname{per}\left(B_{22}\right) \text {. }
$$

This result was later proved by Lieb, see [6], and subsequently generalized to the symmetric algebra by Neuberger, see [1].

The similarity between (1.2) and (1.5) led naturally to the conjecture that

$$
\bar{d}_{X}(B) \leq \operatorname{per}(B)
$$

for each $B \in \mathscr{H}_{n}$; so the permanent function is believed to be the "largest" of the normalized immanents. Despite considerable effort (1.6) is still unresolved. Efforts to prove (1.6) have nevertheless led to the discovery of some interesting theorems, some of which provide information not implied by (1.6). James and Liebeck, see [8], proved that if $X$ is an irreducible character of $S_{n}$ and its associated partition is of the form $\left\{p, q, 1^{r}\right\}, p+q+r=n$, then (1.6) holds for each $B \in \mathscr{H}_{n}$. The author, see [9], proved that $\bar{d}_{c}(B) \leq \operatorname{per}(B)$ for all $B \in \mathscr{H}_{n}$ and all $c \in \mathbb{C} S_{n}$ for which there exists an $f \in \mathbb{C} S_{n}$ and a $\Delta \subset\{1,2, \ldots, n\}$ such that

(1) $c(\sigma)=\sum_{\tau} \overline{f(\sigma \tau)} f(\tau)$ each $\sigma \in S_{n}$,

(2) $\tau f=f$ for each $\tau \in S_{n}$ such that $\tau(\Delta)=\Delta$.

It is easy to show, see [9], that all irreducible characters of $S_{n}$ derived from two-term partitions are expressible as sums of such functions $c$. Hence, this theorem includes the $r=0$ part of the James-Liebeck result as a special case. 
Heyfron, a student of James, see [10], considered the single-hook characters, characters derived from partitions of the form $\left\{q, 1^{n-q}\right\}$, and proved that the associated normalized immanents increase with $q$. In other words, if $X_{1}$ is derived from $\left\{q, 1^{n-q}\right\}$ and $X_{2}$ is derived from $\left\{q+1,1^{n-q-1}\right\}$ then

$$
\bar{d}_{X_{1}}(B) \leq \bar{d}_{X_{2}}(B)
$$

for each $B \in \mathscr{H}_{n}$. This result was originally conjectured by Merris and Watkins, see [11], who proved (1.7) in case $q=0,1$, or $n-1$. It will be shown that our main result generalizes Schur's inequality by replacing (1.3) with a chain of inequalities starting with $\bar{d}_{X}(B)$ and terminating with $\operatorname{det}(B)$. Moreover, our main result reduces Heyfron's result to a special case and, in conjunction with [9], implies and extends the James-Liebeck result.

\section{NOTATION AND BACKGROUND}

We let $V$ denote $\mathbb{C}^{m}$ for a fixed positive integer $m$ which will usually be clear from context. For $n \geq 1$ we let $T_{n}(V)$, often abbreviated to $T_{n}$, denote the set of all $n$-linear complex valued functions on $V$. If $n=0$ then $T_{n}(V)$ denotes $\mathbb{C}$. Given an inner product $\langle$,$\rangle on V$ we extend $\langle$,$\rangle to each of$ the spaces $T_{n}$ by choosing an orthonormal basis $\left\{e_{i}\right\}_{i=1}^{m}$ for $V$ and defining

$$
\langle A, B\rangle=\sum_{q_{1}=1}^{m} \sum_{q_{2}=1}^{m} \ldots \sum_{q_{n}=1}^{m} A\left(e_{q_{1}}, e_{q_{2}}, \ldots, e_{q_{n}}\right) \overline{B\left(e_{q_{1}}, e_{q_{2}}, \ldots, e_{q_{n}}\right)}
$$

for each $A, B \in T_{n}$. Note that this extended $\langle$,$\rangle is independent of the$ orthonormal basis $\left\{\boldsymbol{e}_{i}\right\}_{i=1}^{m}$.

If $A \in T_{n}$ and $B \in T_{p}$ then the tensor product of $A$ and $B$, denoted by $A \otimes B$, is the member of $T_{n+p}$ such that if $x_{1}, x_{2}, \ldots, x_{n+p} \in V$ then

$$
\begin{aligned}
(A \otimes & B \\
\quad & \left(x_{1}, x_{2}, \ldots, x_{n+p}\right) \\
& =A\left(x_{1}, x_{2}, \ldots, x_{n}\right) B\left(x_{n+1}, x_{n+2}, \ldots, x_{n+p}\right) .
\end{aligned}
$$

Note that $\|A \otimes B\|=\|A\| \cdot\|B\|$.

We now define an action of $S_{n}$ on $T_{n}$ which we extend to $\mathbb{C} S_{n}$ in such a way that $T_{n}$ becomes a left $\mathbb{C} S_{n}$ module. If $\sigma \in S_{n}$ and $A \in T_{n}$ then by $\sigma A$ we mean the member of $T_{n}$ defined by

$$
(\sigma A)\left(x_{1}, x_{2}, \ldots, x_{n}\right)=A\left(x_{\sigma(1)}, x_{\sigma(2)}, \ldots, x_{\sigma(n)}\right)
$$

for $x_{1}, x_{2}, \ldots, x_{n} \in V$. Clearly, $(\sigma \tau) A=\sigma(\tau A)$ for $\sigma, \tau \in S_{n}$, and $e A=A$ where $e$ denotes the identity. Since each $\psi \in \mathbb{C} S_{n}$ is represented in the form $\sum_{\sigma \in S_{n}} \psi(\sigma) \sigma$ we define $\psi A$ to be $\sum_{\sigma} \psi(\sigma) \sigma A$. Note that if $A, B \in T_{n}$ then $\langle\sigma A, B\rangle=\left\langle A, \sigma^{-1} B\right\rangle$ for $\sigma \in S_{n}$ and, consequently, if $\psi \in \mathbb{C} S_{n}$ then $\langle\psi A, B\rangle=\left\langle A, \psi^{*} B\right\rangle$ where $\psi^{*}(\sigma)=\overline{\psi\left(\sigma^{-1}\right)}$ for each $\sigma \in S_{n}$.

A member of $A$ of $T_{n}$ is said to be decomposable if there exists $f_{1}, f_{2}, \ldots$, $f_{n} \in V^{*}$, the dual of $V$, such that $A=f_{1} \otimes f_{2} \otimes \cdots \otimes f_{n}$. In this case 
$\|A\|=\prod_{i=1}^{n}\left\|f_{u}\right\|$ and if $\sigma \in S_{n}$ then $\sigma A=f_{\sigma^{-1}(1)} \otimes \cdots \otimes f_{\sigma^{-1}(n)}$ so, if $x_{1}$, $x_{2}, \ldots, x_{n}$ are members of $V$ such that $f_{i}(y)=\left\langle y, x_{i}\right\rangle$ each $y \in V$ and $1 \leq i \leq n$, then $\sigma A=\left(x_{\sigma^{-1}(1)}\right)^{*} \otimes\left(x_{\sigma^{-1}(2)}\right)^{*} \otimes \cdots \otimes\left(x_{\sigma^{-1}(n)}\right)^{*}$ where the conjugate linear map $z \rightarrow z^{*}$ is defined by $z^{*}(y)=\langle y, z\rangle$ for $y, z \in V$. Note that $\langle x, y\rangle=\left\langle y^{*}, z^{*}\right\rangle$. Consequently,

$$
\left\langle x_{1}^{*} \otimes x_{2}^{*} \otimes \cdots \otimes x_{n}^{*}, y_{1}^{*} \otimes y_{2}^{*} \otimes \cdots \otimes y_{n}^{*}\right\rangle=\prod_{i=1}^{n}\left\langle x_{i}^{*}, y_{i}^{*}\right\rangle=\prod_{i=1}^{n}\left\langle y_{i}, x_{i}\right\rangle
$$

for $x_{i}, y_{i} \in V, 1 \leq i \leq n$.

Inequalities involving immanents translate simply into inequalities involving decomposable tensors. Moreover, the converse is also true. The connection between these two types of inequalities is presented in the following:

Lemma 1. If $\psi_{1}, \psi_{2} \in \mathbb{C} S_{n}$ then $d_{\psi_{1}}(B) \leq d_{\psi_{2}}(B)$ for each $B \in \mathscr{H}_{n}$ if and only if $\left\langle\psi_{1} Y, Y\right\rangle \leq\left\langle\psi_{2} Y, Y\right\rangle$ for each decomposable $Y \in T_{n}$.

Proof. If $B \in \mathscr{L}_{n}$ then there exist $y_{1}, y_{2}, \ldots, y_{n} \in V(m \geq n)$ such that $b_{i j}=\left\langle y_{j}, y_{i}\right\rangle, 1 \leq i, j \leq n$. Letting $Y$ denote $y_{1}^{*} \otimes y_{2}^{*} \otimes \cdots \otimes y_{n}^{*}$ we have, for $\psi \in \mathbb{C} S_{n}$,

$$
\begin{aligned}
d_{\psi}(B) & =\sum_{\sigma} \psi(\sigma) \prod_{i=1}^{n} b_{i \sigma(i)}=\sum_{\sigma} \psi(\sigma) \prod_{i=1}^{n}\left\langle y_{\sigma(i)}, y_{i}\right\rangle=\sum_{\sigma} \psi(\sigma) \prod_{i}\left\langle y_{i}, y_{\sigma^{-1}(i)}\right\rangle \\
& =\sum_{\sigma} \psi(\sigma)\left\langle y_{\sigma^{-1}(1)}^{*} \otimes \cdots \otimes y_{\sigma^{-1}(n)}^{*}, y_{1}^{*} \otimes \cdots \otimes y_{n}^{*}\right\rangle \\
& =\sum_{\sigma} \psi(\sigma)\langle\sigma Y, Y\rangle=\langle\psi Y, Y\rangle .
\end{aligned}
$$

Hence, if $\left\langle\psi_{1} Y, Y\right\rangle \leq\left\langle\psi_{2} Y, Y\right\rangle$ for each decomposable $Y \in T_{n}$ then $d_{\psi_{1}}(B) \leq$ $d_{\psi_{2}}(B)$ for each $B \in T_{n}$. The proof of the converse is similar, and omitted.

When dealing with the characters of the symmetric group our notation follows Marcus, see [7, Chapter 6]. If $\alpha=\left\{\alpha_{1}, \alpha_{2}, \ldots, \alpha_{t}\right\}$ is a partition of $n$, we always assume $\alpha_{1} \geq \alpha_{2} \geq \cdots \geq \alpha_{t}$, and $\varphi \in S_{n}$ then by $D_{\alpha, \varphi}$ we mean the Young diagram whose first row contains $\varphi(1), \varphi(2), \ldots, \varphi\left(\alpha_{1}\right)$, whose second row contains $\varphi\left(\alpha_{1}+1\right), \varphi\left(\alpha_{1}+2\right), \ldots, \varphi\left(\alpha_{1}+\alpha_{2}\right)$ etc. By $R_{\alpha, \varphi}$ and $C_{\alpha, \varphi}$ we mean, respectively, the row and column groups of $D_{\alpha, \varphi}$, and by $r_{\alpha, \varphi}$ and $c_{\alpha, \varphi}$ we mean the corresponding row and column symmetrizers. Explicitly, $r_{\alpha, \varphi}=\sum \sigma$ where summation is over $R_{\alpha, \varphi}$, and $c_{\alpha, \varphi}=\sum \varepsilon(\sigma) \sigma$ where the summation is over $C_{\alpha, \varphi}$, so it is perhaps more accurate to refer to $c_{\alpha, \varphi}$ as a column skew-symmetrizer. The Young symmetrizer $\mathscr{E}_{\alpha, \varphi}$, associated with $\alpha$ and $\varphi$, is then $r_{\alpha, \varphi} c_{\alpha, \varphi}$. We denote the irreducible character associated with $\alpha$ by $X_{\alpha}$.

We require a formula for $X_{\alpha}$ in terms of the associated Young symmetrizers. According to [12, Theorem 1, p. 108], we have

$$
X_{\alpha}(\sigma)=\frac{n_{\alpha}}{n !} \sum_{g \in S_{n}} \mathscr{E}_{\alpha, \varphi}\left(g^{-1} \sigma^{-1} g\right)
$$


where $n_{\alpha}$ is the dimension of the representation which is simply $X_{\alpha}(e)$. For $f \in \mathbb{C} S_{n}$ we let $\hat{f}=(f(e))^{-1} f, f(e) \neq 0$, so, since $n_{\alpha}=X_{\alpha}(e)$, we have

$$
\widehat{X}_{\alpha}(\sigma)=(n !)^{-1} \sum_{g} \mathscr{E}_{\alpha, \varphi}\left(g^{-1} \sigma^{-1} g\right)
$$

but $X_{\alpha}$ and $\widehat{X}_{\alpha}$ are real-valued class functions so $\widehat{X}_{\alpha}\left(\sigma^{-1}\right)=\widehat{X}_{\alpha}(\sigma)$ and

$$
\widehat{X}_{\alpha}(\sigma)=(n !)^{-1} \sum_{g} \mathscr{E}_{\alpha, \varphi}\left(g^{-1} \sigma g\right)
$$

which implies that

$$
\widehat{X}_{\alpha}=(n !)^{-1} \sum_{\sigma} \sigma r_{\alpha, \varphi} c_{\alpha, \varphi} \sigma^{-1}
$$

for each $\varphi \in S_{n}$. The multiplication $\sigma \tau_{\alpha, \varphi}$ in (2.4) involves a slight abuse of language since $\sigma \in S_{n}$ and $r_{\alpha, \varphi} \in \mathbb{C} S_{n}$. In such cases we identify $\sigma$ with the member of $\mathbb{C} S_{n}$ that assumes the value 1 at $\sigma$ and 0 elsewhere.

As is clear from (2.1) the character $X_{\alpha}$ is independent of $\varphi$ and, consequently, $\varphi$ may be chosen according to convenience. Since $\varphi$ will always be clear from context we delete $\varphi$ from the notation and abbreviate as follows: $R_{\alpha}=R_{\alpha, \varphi}, C_{\alpha}=C_{\alpha, \varphi}, r_{\alpha}=r_{\alpha, \varphi}, c_{\alpha}=c_{\alpha, \varphi}, \mathscr{E}_{\alpha}=\mathscr{E}_{\alpha, \varphi}$, and $D_{\alpha}=D_{\alpha, \varphi}$.

Lemma 2. If $\psi, \mathscr{E} \in \mathbb{C} S_{n}$ and $\mathscr{E}^{2}=k \mathscr{E}$ for some $k \neq 0$ then

$$
\sum_{\sigma} \sigma \psi \mathscr{E} \sigma^{-1}=\sum_{\sigma} \sigma \mathscr{E} \psi \sigma^{-1}=k^{-1} \sum_{\sigma} \sigma \mathscr{E} \psi \mathscr{E} \sigma^{-1} .
$$

Proof. If $f \in \mathbb{C} S_{n}$ and $\tau \in S_{n}$ then, by setting $u=\tau \sigma^{-1}$, one sees that

$$
\sum_{\sigma} \sigma f \tau \sigma^{-1}=\sum_{u} u^{-1} \tau f u=\sum_{\sigma} \sigma \tau f \sigma^{-1},
$$

an equality that immediately implies that if $f, g \in \mathbb{C} S_{n}$ then

$$
\sum_{\sigma} \sigma f g \sigma^{-1}=\sum_{\sigma} \sigma g f \sigma^{-1} \text {. }
$$

Consequently, we have

$$
\sum_{\sigma} \sigma \psi \mathscr{E} \sigma^{-1}=k^{-1} \sum_{\sigma} \sigma \psi \mathscr{E}^{2} \sigma^{-1}=k^{-1} \sum_{\sigma} \sigma \mathscr{E} \psi \mathscr{E} \sigma^{-1}
$$

as required.

Combining Lemma 2 with formula (2.4) we see that

$$
\widehat{X}_{\alpha}=\left(n !\left|R_{\alpha}\right|\right)^{-1} \sum_{\sigma} \sigma r_{\alpha} c_{\alpha} r_{\alpha} \sigma^{-1}
$$

and

$$
\widehat{X}_{\alpha}=\left(n !\left|C_{\alpha}\right|\right)^{-1} \sum_{\sigma} \sigma c_{\alpha} r_{\alpha} c_{\alpha} \sigma^{-1}
$$

for each $\varphi \in S_{n}$. 
If $G$ is a finite group, and $X$ is a character of $H$ then $X^{\uparrow}$, the character of $G$ induced from $X$, is defined by

$$
X^{\dagger}(u)=|H|^{-1} \sum X\left(\sigma^{-1} u \sigma\right)
$$

where summation is over all $\sigma \in G$ such that $\sigma^{-1} u \sigma \in H$. See [13, p. 30], for details. Extending $X \in \mathbb{C} H$ to all of $G$ by defining $X(\sigma)=0$ for $\sigma \in G-H$ we see that $(2.8)$ immediately implies that

$$
X^{\uparrow}=|H|^{-1} \sum_{\sigma \in G} \sigma X \sigma^{-1}
$$

and, since $X^{\uparrow}(e)=X(e)|G| /|H|$,

$$
\widehat{X^{\dagger}}=(X(e)|G|)^{-1} \sum_{\sigma \in G} \sigma X \sigma^{-1} .
$$

If $\Delta \subset\{1,2, \ldots, n\}$ then by $G(\Delta)$ we mean the set of all $\sigma \in S_{n}$ such that $\sigma(\Delta)=\Delta$ and $\sigma(i)=i$ if $i \notin \Delta$. The idempotents $\mathscr{S}(\Delta)$ and $\mathscr{A}(\Delta)$ are defined to be $|G(\Delta)|^{-1} \sum_{\sigma \in G(\Delta)} \sigma$ and $|G(\Delta)|^{-1} \sum_{\sigma \in G(\Delta)} \varepsilon(\sigma) \sigma$ respectively. These idempotents provide necessary factorizations of the symmetrizers $r_{\alpha}$ and $c_{\alpha}$, for if the row sets of $D_{\alpha}$ are $\Delta_{1}, \Delta_{2}, \ldots, \Delta_{s}$ and the column sets are $\Gamma_{1}$, $\Gamma_{2}, \ldots, \Gamma_{t}$ then

$$
r_{\alpha}=\prod_{i=1}^{s}\left|G\left(\Delta_{i}\right)\right| \mathscr{S}\left(\Delta_{i}\right)
$$

and

$$
c_{\alpha}=\prod_{j=1}^{t}\left|G\left(\Gamma_{j}\right)\right| \mathscr{A}\left(\Gamma_{j}\right) .
$$

All subsets $\Delta \subset\{1,2, \ldots, N\}$ with $|\Delta|=n$ give rise to groups $G(\Delta)$ isomorphic to $S_{n}$. For our computations we require a definite isomorphism $\sigma \rightarrow \sigma^{\wedge}$ from $G(\Delta)$ to $S_{n}$, so we let $\delta$ be the unique increasing function from $\{1,2, \ldots, n\}$ onto $\Delta$ and define $\sigma^{\wedge}=\delta^{-1} \sigma \delta$ for $\sigma \in G(\Delta)$. Given two disjoint subsets $\Delta_{1}$ and $\Delta_{2}$ of $\{1,2, \ldots, N\}$ with $\left|\Delta_{1}\right|=n$ and $\left|\Delta_{2}\right|=p$ we require two isomorphisms, " $\wedge$ " and " $\vee$," each as described above. Letting $H$ denote $G\left(\Delta_{1}\right) \cdot G\left(\Delta_{2}\right)$ the map $\gamma \rightarrow\left(\gamma^{\wedge}, \gamma^{\vee}\right)$ is then an isomorphism between $H$ and $S_{n} \oplus S_{p}$.

To define $\mathbb{C S}_{n} \otimes C S_{p}$ we consider the special case $\Delta_{1}=\{1,2, \ldots, n\}$ and $\Delta_{2}=\{n+1, n+2, \ldots, n+p\}$ and require that $\sigma \otimes \tau, \sigma \in S_{n}$ and $\tau \in S_{p}$, denote the member of $\mathbb{C} H$ such that $(\sigma \otimes \tau)(\theta)=1$ or 0 depending on whether $\sigma=\theta^{\wedge}$ and $\tau=\theta^{\vee}$, or not. Then, we extend $\otimes$ to the rest of $\mathbb{C} S_{n} \times \mathbb{C} S_{p}$ by requiring

$$
f \otimes g=\sum_{\sigma \in S_{n}} \sum_{\tau \in S_{p}} f(\sigma) g(\tau) \sigma \otimes \tau .
$$


In this way $\mathbb{C} S_{n} \otimes \mathbb{C} S_{p}$ is identified with a subalgebra of $\mathbb{C} S_{n+p}$, namely $\mathbb{C} H$.

There are certain simple properties satisfied by " $\wedge$ ", “ $\vee$ ", and $\otimes$ which we present without proof:

Lemma 3. Suppose $\Delta_{1}=\{1,2, \ldots, n\}, \Delta_{2}=\{n+1, n+2, \ldots, n+p\}$, $H=G\left(\Delta_{1}\right) \cdot G\left(\Delta_{2}\right), \sigma \in H, f \in \mathbb{C} S_{n}, g \in \mathbb{C S}_{p}, A \in T_{n}$, and $B \in T_{p}$. Then,

(1) $\sigma(f \otimes g)=\sigma^{\wedge} f \otimes \sigma^{\vee} g$,

(2) $(f \otimes g) \sigma=f \sigma^{\wedge} \otimes g \sigma^{\vee}$,

(3) $\sigma(A \otimes B)=\left(\sigma^{\wedge} A\right) \otimes\left(\sigma^{\vee} B\right)$,

(4) $(f \otimes g)(A \otimes B)=(f A) \otimes(g B)$.

Returning now to the context of formulas (2.8), (2.9), and (2.10) we see that the map $X \rightarrow X^{\dagger}$ need not be restricted to characters $X$ of $H$. Indeed, we may define $f^{\dagger}$ by formula (2.8) or (2.9) for any $f \in \mathbb{C} H$. Consequently, we state the following:

Lemma 4. Suppose $f \in \mathbb{C} S_{n}$ and $g \in \mathbb{C} S_{p}$ are class functions such that $f(e)=$ $g(e)=1$. Then,

$$
(\widehat{f \otimes g})^{\uparrow}=\left(\begin{array}{c}
n+p \\
n
\end{array}\right)^{-1} \sum_{\varphi \in U} \varphi(f \otimes g) \varphi^{-1}
$$

where $U$ denotes the set of all members $\varphi$ of $S_{n+p}$ such that $\varphi$ restricted to $\{1,2, \ldots, n\}$ is increasing and $\varphi$ restricted to $\{n+1, n+2, \ldots, n+p\}$ is increasing.

Proof. Let $H=G(\{1,2, \ldots, n\}) \cdot G(\{n+1, n+2, \ldots, n+p\})$ and note that $U$ is a set of distinct representatives of the left cosets of $H$ in $S_{n+p}$. The rest of the proof is now a straightforward computation:

$$
\begin{aligned}
(n+p) !(\widehat{f \otimes g})^{\uparrow} & =\sum_{\sigma} \sigma(f \otimes g) \sigma^{-1}=\sum_{\tau \in H} \sum_{\varphi \in U} \varphi \tau(f \otimes g) \tau^{-1} \varphi^{-1} \\
& =\sum_{\tau \in H} \sum_{\varphi \in U} \varphi\left(\tau^{\wedge} f\left(\tau^{\wedge}\right)^{-1} \otimes \tau^{\vee} g\left(\tau^{\vee}\right)^{-1}\right) \varphi^{-1} \\
& =|H| \sum_{\varphi \in U} \varphi(f \otimes g) \varphi^{-1},
\end{aligned}
$$

where the penultimate expression is obtained from its predecessor using Lemma 3. Since $|H|=n ! p !$, the proof is complete.

\section{MAin RESUlts}

If $f \in \mathbb{C} S_{n}$ then we shall write $f \geq 0$ if $\langle f Y, Y\rangle \geq 0$ for each decomposable $Y \in T_{n}$. Hence, $f \leq g$ if $\langle f Y, Y\rangle \leq\langle g Y, Y\rangle$ for each decomposable $Y \in T_{n}$. Our main results are Theorems 3 and 6 . Theorem 3 provides a stepping-up inequality: it shows how, given an irreducible character $\psi$ of $S_{n}$, to find a second character $\mathscr{E}$, induced from a Young subgroup of $S_{n}$, such that $\psi \leq \mathscr{E}$. 
In the same sense Theorem 6 provides a stepping down inequality. We combine Theorems 3 and 6 to obtain Theorem 7, which is the result mentioned in the abstract.

If $k \geq 1$ then $\Lambda^{k}(V)$ denotes the set of all alternating $k$-linear functions on $V$, and, if $A \in \Lambda^{n}(V)$ and $B \in \Lambda^{p}(V)$, then $A \wedge B$ denotes $\mathscr{A}(\Delta)(A \otimes B)$ where $\Delta=\{1,2, \ldots, n+p\}$. The following appears in [14].

Theorem 1. If $A \in \Lambda^{n}(V), B \in \Lambda^{p}(B)$, and either $A$ or $B$ is decomposable then

$$
\|A \wedge B\|^{2} \leq\left(\begin{array}{c}
n+p \\
n
\end{array}\right)^{-1}\|A\|^{2}\|B\|^{2} .
$$

In the context of Theorem 1 decomposability means decomposability with respect to the multiplication in the exterior algebra, so the statement that $A \in$ $\Lambda^{n}(V)$ is decomposable means that there exists $x_{1}, x_{2}, \ldots, x_{n} \in V$ such that $A=x_{1}^{*} \wedge x_{2}^{*} \wedge \cdots \wedge x_{n}^{*}$. Our purposes require a strengthening of Theorem 1 .

Theorem 2. If $A$ is a decomposable member of $T_{n}, \Delta_{1}=\{1,2, \ldots, n\}, \Delta_{2} \subset$ $\{n+1, n+2, \ldots, n+p+r\}$ with $\left|\Delta_{2}\right|=p$, and $\Delta$ denotes $\Delta_{1} \cup \Delta_{2}$ then

$$
\|\mathscr{A}(\Delta)(A \otimes B)\|^{2} \leq\left(\begin{array}{c}
n+p \\
n
\end{array}\right)^{-1}\left\|\left(\mathscr{A}\left(\Delta_{1}\right)\right)^{\wedge} A\right\|^{2}\left\|\left(\mathscr{A}\left(\Delta_{2}\right)\right)^{\vee} B\right\|^{2}
$$

for each $B \in T_{p+r}$.

Proof. First, note that it is permissible to assume $\Delta_{2}=\{n+1, n+2, \ldots, n+p\}$. Let $\left\{e_{i}\right\}_{i=1}^{m}$ be an orthonormal basis for $V$. Then,

$$
\begin{aligned}
\|\mathscr{A}(\Delta)(A \otimes B)\|^{2} & =\sum_{q \in \Gamma_{n+p+r, m}}\left|\mathscr{A}(\Delta)(A \otimes B)\left(e_{q}\right)\right|^{2} \\
& =\sum_{q \in \Gamma_{n+p, m}} \sum_{t \in \Gamma_{r, m}}\left|\mathscr{A}(\Delta)(A \otimes B)\left(e_{q}, e_{t}\right)\right|^{2}
\end{aligned}
$$

where:

(a) for positive integers $s, t, \Gamma_{s, t}$ denotes all sequences of length $s$ each of whose terms is a member of $\{1,2, \ldots, t\}$,

(b) $\mathscr{A}(\Delta)(A \otimes B)\left(e_{q}\right)$ denotes $\mathscr{A}(\Delta)(A \otimes B)\left(e_{q_{1}}, e_{q_{2}}, \ldots, e_{q_{n+p+r}}\right)$ for $q \in$ $\Gamma_{n+p+r, m}$, and

(c) $\mathscr{A}(\Delta)(A \otimes B)\left(e_{q}, e_{t}\right)$ denotes

$$
\mathscr{A}(\Delta)(A \otimes B)\left(e_{q_{1}}, e_{q_{2}}, \ldots, e_{q_{n+p}}, e_{t_{1}}, e_{t_{2}}, \ldots, e_{t_{r}}\right)
$$

for $q \in \Gamma_{n+p, m}$ and $t \in \Gamma_{r, m}$.

Since $\mathscr{A}(\Delta)$ does not effect the last $r$ places of $B$ we let $B_{t}, t \in \Gamma_{r, m}$, denote the member of $T_{p}$ such that if $z_{1}, z_{2}, \ldots, z_{p}$ are in $V$ then

$$
B_{t}\left(z_{1}, z_{2}, \ldots, z_{p}\right)=B\left(z_{1}, z_{2}, \ldots, z_{p}, e_{t_{1}}, e_{t_{2}}, \ldots, e_{t_{r}}\right)
$$

and note that

$$
\mathscr{A}(\Delta)(A \otimes B)\left(e_{q}, e_{t}\right)=\mathscr{A}(\Delta)\left(A \otimes B_{t}\right)\left(e_{q}\right)
$$


for each $q \in \Gamma_{n+p, m}$. Hence,

$$
\begin{aligned}
\|\mathscr{A}(\Delta)(A \otimes B)\|^{2} & =\sum_{t \in \Gamma_{r, m}} \sum_{q \in \Gamma_{n+p, m}}\left|\mathscr{A}(\Delta)\left(A \otimes B_{t}\right)\left(e_{q}\right)\right|^{2} \\
& =\sum_{t \in \Gamma_{r, m}}\left\|\mathscr{A}(\Delta)\left(A \otimes B_{t}\right)\right\|^{2} .
\end{aligned}
$$

Now, by Theorem 1 ,

$$
\left\|\mathscr{A}(\Delta)\left(\mathscr{A}\left(\Delta_{1}\right)^{\wedge} A \otimes \mathscr{A}\left(\Delta_{2}\right)^{\vee} B_{t}\right)\right\|^{2} \leq\left(\begin{array}{c}
n+p \\
n
\end{array}\right)^{-1}\left\|\mathscr{A}\left(\Delta_{1}\right)^{\wedge} A\right\|^{2}\left\|\mathscr{A}\left(\Delta_{2}\right)^{\vee} B\right\|^{2} .
$$

But, $\mathscr{A}(\Delta) \sigma=\sigma \mathscr{A}(\Delta)=\varepsilon(\sigma) \mathscr{A}(\Delta)$ for each $\sigma \in G(\Delta)$. Hence, $\mathscr{A}(\Delta) \mathscr{A}\left(\Delta_{i}\right)=$ $\mathscr{A}(\Delta), i=1,2$, and

$$
\begin{aligned}
\mathscr{A}(\Delta)\left(\mathscr{A}\left(\Delta_{1}\right)^{\wedge} A \otimes \mathscr{A}\left(\Delta_{2}\right)^{\vee} B_{t}\right) & =\mathscr{A}(\Delta) \mathscr{A}\left(\Delta_{1}\right) \mathscr{A}\left(\Delta_{2}\right)\left(A \otimes B_{t}\right) \\
& =\mathscr{A}(\Delta)\left(A \otimes B_{t}\right) .
\end{aligned}
$$

Therefore

$$
\begin{aligned}
\|\mathscr{A}(\Delta)(A \otimes B)\|^{2} & =\sum_{t \in \Gamma_{r, m}}\left\|\mathscr{A}(\Delta)\left(A \otimes B_{t}\right)\right\|^{2} \\
& \leq\left(\begin{array}{c}
n+p \\
n
\end{array}\right)^{-1} \sum_{t \in \Gamma_{r, m}}\left\|\mathscr{A}\left(\Delta_{1}\right)^{\wedge} A\right\|^{2}\left\|\mathscr{A}\left(\Delta_{2}\right)^{\vee} B_{t}\right\|^{2} \\
& =\left(\begin{array}{c}
n+p \\
n
\end{array}\right)^{-1}\left\|\mathscr{A}\left(\Delta_{1}\right)^{\wedge} A\right\|^{2} \cdot \sum_{t \in \Gamma_{r, m}}\left\|\mathscr{A}\left(\Delta_{2}\right)^{\vee} B_{t}\right\|^{2} \\
& =\left(\begin{array}{c}
n+p \\
n
\end{array}\right)^{-1}\left\|\mathscr{A}\left(\Delta_{1}\right)^{\wedge} A\right\|^{2}\left\|\mathscr{A}\left(\Delta_{2}\right)^{\vee} B\right\|^{2} .
\end{aligned}
$$

Given $Y=y_{1}^{*} \otimes y_{2}^{*} \otimes \cdots \otimes y_{N}^{*}$ where $y_{1}, y_{2}, \ldots, y_{N} \in V$ and $n<N$ we shall need to express $Y$ as $Y_{1, n} \otimes Y_{2, n}$ where $Y_{1, n}=y_{1}^{*} \otimes y_{2}^{*} \otimes \cdots \otimes y_{N-n}^{*}$ and $Y_{2, n}=y_{N-n+1}^{*} \otimes y_{N-n+2}^{*} \otimes \cdots \otimes y_{N}^{*}$. Since $n$ will usually be clear from context we write $Y_{1}$ instead of $Y_{1, n}$, and $Y_{2}$ instead of $Y_{2, n}$. Moreover, if $\sigma \in S_{n+p}$ then $Y_{1}(\sigma)$ denotes $y_{\sigma(1)}^{*} \otimes y_{\sigma(2)}^{*} \otimes \cdots \otimes y_{\sigma(N-n)}^{*}$ and $Y_{2}(\sigma)$ denotes $y_{\sigma(N-n+1)}^{*} \otimes y_{\sigma(N-n+2)}^{*} \otimes \cdots \otimes y_{\sigma(N)}^{*}$. Given these definitions it is easy to see that if $\sigma \in S_{n+p}$ then $\sigma^{-1} Y=Y_{1}(\sigma) \otimes Y_{2}(\sigma)$, and if $\tau \in H=G\left(\Delta_{1}\right) \cdot G\left(\Delta_{2}\right)$, where $\Delta_{1}=\{1,2, \ldots, N-n\}$ and $\Delta_{2}=\{N-n+1, N-n+2, \ldots, N\}$, then

$$
\begin{aligned}
(\sigma \tau)^{-1} Y & =\tau^{-1}\left(\sigma^{-1} Y\right) \\
& =\left(\left(\tau^{\wedge}\right)^{-1} \otimes\left(\tau^{\vee}\right)^{-1}\right)\left(Y_{1}(\sigma) \otimes Y_{2}(\sigma)\right) \\
& =\left(\tau^{-1}\right)^{\wedge} Y_{1}(\sigma) \otimes\left(\tau^{-1}\right)^{\vee} Y_{2}(\sigma) .
\end{aligned}
$$

Theorem 3. If $\alpha=\left\{\alpha_{1}, \alpha_{2}, \ldots \alpha_{n+p}\right\}=\left\{\alpha_{1}, \alpha_{2}, \ldots, \alpha_{p}, 1^{n}\right\}$ is a partition of $N, \beta=\left\{\beta_{1}, \beta_{2}, \ldots, \beta_{t}\right\}$, it is associated (or transpose) partition, and $\alpha^{\prime}$ 
denotes $\left\{\alpha_{1}, \alpha_{2}, \ldots, \alpha_{p}\right\}$ then

$$
\left\langle\widehat{X}_{\alpha} Y, Y\right\rangle \leq\left(\begin{array}{c}
N \\
n
\end{array}\right)^{-1} \sum_{\varphi \in U}\left\langle\widehat{X}_{\alpha^{\prime}} Y_{1}(\varphi), Y_{1}(\varphi)\right\rangle\left\langle\widehat{X}_{\left\{1^{n}\right\}} Y_{2}(\varphi), Y_{2}(\varphi)\right\rangle
$$

for each decomposable $Y \in T_{N}$, where $U$ denotes the set of all members of $S_{N}$ that increase on $\{1,2, \ldots, N-n\}$ and $\{N-n+1, N-n+2, \ldots, N\}$. Consequently,

Proof. By (2.4) we have

$$
\widehat{X}_{\alpha} \leq\left(\widehat{\widehat{X}}_{\alpha^{\prime}} \otimes \widehat{X}_{\left\{1^{n}\right\}}\right)^{\uparrow} .
$$

$$
\widehat{X}_{\alpha}=(N !)^{-1} \sum_{\sigma \in S_{N}} \sigma r_{\alpha} c_{\alpha} \sigma^{-1}
$$

for any $\varphi \in S_{N}$. In the following the underlying Young diagram is the natural Young diagram obtained by setting $\varphi=e$. Moreover, we abbreviate $r_{\alpha}$ with $r$ and $c_{\alpha}$ with $c$. We let $c^{\prime}$ denote $(n+p) ! \mathscr{A}\left(\Delta_{1}\right)$ and $c^{\prime \prime}$ denote $\prod_{j=2}^{t}\left|G\left(\Delta_{j}\right)\right| \mathscr{A}\left(\Delta_{j}\right)$ where $\Delta_{1}, \Delta_{2}, \ldots, \Delta_{t}$ are the column sets of $D_{\alpha}$. Note that $c^{\prime}$ is the symmetrizer associated with the first column of $D_{\alpha}$ and $c^{\prime \prime}$ is the product of the symmetrizers associated with the other columns. Hence, $c=c^{\prime} c^{\prime \prime}=c^{\prime \prime} c^{\prime}$. Now, by (2.6), we have

$$
\begin{aligned}
N ! \widehat{X}_{\alpha} & =\left|R_{\alpha}\right|^{-1} \sum_{\sigma} \sigma r c r \sigma^{-1}=\left|R_{\alpha}\right|^{-1} \sum_{\sigma} \sigma r c^{\prime} c^{\prime \prime} r \sigma^{-1} \\
& =(n+p) !\left(\left|R_{\alpha} \| C_{\alpha}\right|\right)^{-1} \sum_{\sigma} \sigma r c^{\prime}\left(c^{\prime \prime}\right)^{2} r \sigma^{-1} \\
& =(n+p) !\left(\left|R_{\alpha}\right|\left|C_{\alpha}\right|\right)^{-1} \sum_{\sigma} \sigma r c^{\prime \prime} c^{\prime} c^{\prime \prime} r \sigma^{-1}
\end{aligned}
$$

since $(n+p) !\left(c^{\prime \prime}\right)^{2}=\left|C_{\alpha}\right| c^{\prime \prime}$. Let $K=(n+p) !\left(\left|R_{\alpha}\right|\left|C_{\alpha}\right| N !\right)^{-1}$. Now,

$$
\begin{aligned}
\left\langle\widehat{X}_{\alpha} Y, Y\right\rangle & =K \sum_{\alpha}\left\langle\sigma r c^{\prime \prime} c^{\prime} c^{\prime \prime} r \sigma^{-1} Y, Y\right\rangle \\
& =K \sum_{\sigma}\left\langle c^{\prime}\left(c^{\prime \prime} r \sigma^{-1} Y\right), c^{\prime \prime} r \sigma^{-1} Y\right\rangle \\
& =K \sum_{\sigma}\left\langle c^{\prime}\left(c^{\prime \prime} r\left(Y_{1}(\sigma) \otimes Y_{2}(\sigma)\right)\right), c^{\prime \prime} r\left(Y_{1}(\sigma) \otimes Y_{2}(\sigma)\right)\right\rangle \\
& =K \sum_{\sigma}\left\langle c^{\prime}\left(\left(c^{\prime \prime} r\right)^{\wedge} Y_{1}(\sigma) \otimes Y_{2}(\sigma)\right),\left(c^{\prime \prime} r\right)^{\wedge} Y_{1}(\sigma) \otimes Y_{2}(\sigma)\right\rangle \\
& =(n+p) ! K \sum_{\sigma}\left\|\mathscr{A}\left(\Delta_{1}\right)\left(\left(c^{\prime \prime} r\right)^{\wedge} Y_{1}(\sigma) \otimes Y_{2}(\sigma)\right)\right\|^{2} \\
& \leq(n+p) !\left(\begin{array}{c}
n+p \\
n
\end{array}\right)^{-1} K \sum_{\sigma}\left\|\mathscr{A}\left(\Delta^{\prime}\right)\left(\left(c^{\prime \prime} r\right)^{\wedge} Y_{1}(\sigma)\right)\right\|^{2}\left\|\mathscr{A}\left(\Delta_{0}\right) Y_{2}(\sigma)\right\|^{2}
\end{aligned}
$$

by Theorem 2, where $\Delta^{\prime}=\Delta_{1}-\{N-n+1, N-n+2, \ldots, N\}$ and $\Delta_{0}=$ $\{1,2, \ldots, n\}$. Letting $\mathscr{U}$ be as in the statement of the theorem and observing 
that $\mathscr{A}\left(\Delta^{\prime}\right) c^{\prime \prime}=(p !)^{-1} c_{\alpha^{\prime}}$ and $r_{\alpha}=r_{\alpha^{\prime}}$ the above is

$$
\begin{aligned}
& =n ! p ! K \sum_{\sigma}\left\|(p !)^{-1} c_{\alpha^{\prime}} r_{\alpha^{\prime}} Y_{1}(\sigma)\right\|^{2}\left\|\mathscr{A}\left(\Delta_{0}\right) Y_{2}(\alpha)\right\|^{2} \\
& =n !(p !)^{-1} K \sum_{\varphi \in \mathscr{U}} \sum_{\tau \in H}\left\|c_{\alpha^{\prime}} r_{\alpha^{\prime}} Y_{1}(\varphi \tau)\right\|^{2}\left\|\mathscr{A}\left(\Delta_{0}\right) Y_{2}(\varphi \tau)\right\|^{2} \\
& =n !(p !)^{-1} K \sum_{\varphi \in \mathscr{U}} \sum_{\tau \in H}\left\|c_{\alpha^{\prime}} r_{\alpha^{\prime}}\left(\tau^{\wedge}\right)^{-1} Y_{1}(\varphi)\right\|^{2}\left\|\mathscr{A}\left(\Delta_{0}\right)\left(\tau^{\vee}\right)^{-1} Y_{2}(\varphi)\right\|^{2} \\
& =n !(p !)^{-1} K \sum_{\varphi} \sum_{u \in S_{N-n}} \sum_{\theta \in S_{n}}\left\|c_{\alpha^{\prime}} r_{\alpha^{\prime}} u^{-1} Y_{1}(\varphi)\right\|^{2}\left\|\mathscr{A}\left(\Delta_{0}\right) \theta^{-1} Y_{2}(\varphi)\right\|^{2} \\
& =n !(p !)^{-1} K \sum_{\varphi} \sum_{u, \theta}\left\langle c_{\alpha^{\prime}} r_{\alpha^{\prime}} u^{-1} Y_{1}(\varphi), c_{\alpha^{\prime}} r_{\alpha^{\prime}} u^{-1} Y_{1}(\varphi)\right\rangle\left\|\mathscr{A}\left(\Delta_{0}\right) \theta^{-1} Y_{2}(\varphi)\right\|^{2} \\
& =n !(p !)^{-1}\left|C_{\alpha^{\prime}}\right| K \sum_{\varphi} \sum_{u, \theta}\left\langle\left(u r_{\alpha^{\prime}} c_{\alpha^{\prime}} r_{\alpha^{\prime}} u^{-1}\right) Y_{1}(\varphi), Y_{1}(\varphi)\right\rangle \\
& \times\left\langle\theta \mathscr{A}\left(\Delta_{0}\right) \theta^{-1} Y_{2}(\varphi), Y_{2}(\varphi)\right\rangle
\end{aligned}
$$

But $\theta \mathscr{A}\left(\Delta_{0}\right) \theta^{-1}=\mathscr{A}\left(\Delta_{0}\right)$ for each $\theta \in S_{n}$ and

$$
\sum_{\mu} \mu r_{\alpha^{\prime}} c_{\alpha^{\prime}} r_{\alpha^{\prime}} \mu^{-1}=\left|R_{\alpha^{\prime}}\right| \sum_{\mu} \mu r_{\alpha^{\prime}} c_{\alpha^{\prime}} \mu^{-1}
$$

by Lemma 2 . Since $\left|R_{\alpha^{\prime}}\right|=\left|R_{\alpha}\right|,\left|C_{\alpha^{\prime}}\right|=p !\left|C_{\alpha}\right|((n+p) !)^{-1}$, and $\sum_{\mu} \mu r_{\alpha^{\prime}} c_{\alpha^{\prime}} \mu^{-1}$ $=(N-n) ! \widehat{X}_{\alpha^{\prime}}$ by $(2.4)$, the above is

$$
\begin{aligned}
& =n !\left|R_{\alpha} \| C_{\alpha}\right|(N-n) ! K((n+p) !)^{-1} \sum_{\varphi}\left\langle\widehat{X}_{\alpha^{\prime}} Y_{1}(\varphi), Y_{1}(\varphi)\right\rangle\left\langle\widehat{X}_{\left\{1^{n}\right\}} Y_{2}(\varphi), Y_{2}(\varphi)\right\rangle \\
& =\left(\begin{array}{c}
N \\
n
\end{array}\right)^{-1} \sum_{\varphi}\left\langle\widehat{X}_{\alpha^{\prime}} Y_{1}(\varphi), Y_{1}(\varphi)\right\rangle\left\langle\widehat{X}_{\left\{1^{n}\right\}} Y_{2}(\varphi), Y_{2}(\varphi)\right\rangle
\end{aligned}
$$

which, finally, is the expression appearing in the statement of the theorem. That

$$
\widehat{X}_{\alpha} \leq\left(\widehat{\widehat{X}}_{\alpha^{\prime}} \otimes \widehat{X}_{\left\{1^{n}\right\}}\right)^{\uparrow}
$$

now follows from Lemma 4.

We now present the notation necessary to translate Theorem 3 into the language of matrix theory.

If $s \leq n$ then $Q_{s, n}$ will denote the set of all strictly increasing sequences of length $s$ each of whose terms is a member of $\{1,2, \ldots, n\}$. If $\varphi \in Q_{s, n}$ then the sequence complementary to $\varphi$, denoted by $\varphi^{c}$, is the member of $Q_{n-s, n}$ whose range is complementary to the range of $\varphi$. If $C=\left[c_{i j}\right]$ is an $s \times t$ matrix, $\mu \in Q_{s_{0}, s}, \theta \in Q_{t_{0}, t}$ then $C[\mu \mid \theta]$ denotes the $s_{0} \times t_{0}$ matrix whose $i j$ th term is $c_{\mu(i), \theta(j)}$. Similarly, $C(\mu \mid \varphi)$ denotes the $\left(s-s_{0}\right) \times\left(t-t_{0}\right)$ matrix whose $i j$ th term is $c_{\mu^{c}(i), \theta^{c}(j)}$. 
Now, if $C=\left[c_{i j}\right]=\left[\left(\left\langle y_{j}, y_{i}\right\rangle\right] \in \mathscr{H}_{N}\right.$, then by Theorem 3 and the proof of Lemma 1 we have

$$
\begin{aligned}
\bar{d}_{X_{\alpha}}(C) & =\left\langle\widehat{X}_{\alpha} Y, Y\right\rangle \\
& \leq\left(\begin{array}{c}
N \\
n
\end{array}\right)^{-1} \sum_{\varphi \in \mathscr{U}}\left\langle\widehat{X}_{\alpha^{\prime}} Y_{1}(\varphi), Y_{1}(\varphi)\right\rangle\left\langle\widehat{X}_{\left\{1^{n}\right\}} Y_{2}(\varphi), Y_{2}(\varphi)\right\rangle .
\end{aligned}
$$

But, $\varphi \in \mathscr{U}$ restricted to $\{1,2, \ldots, N-n\}$ is a member of $Q_{N-n, N}$ and $\left\langle\widehat{X}_{\left\{1^{n}\right\}} Y_{2}(\varphi), Y_{2}(\varphi)\right\rangle=\operatorname{det}(C(\varphi \mid \varphi))$. Hence, we have the following:

Theorem 4. If $C=\left[c_{i j}\right] \in \mathscr{H}_{N}, \alpha=\left\{\alpha_{1}, \alpha_{2}, \ldots, \alpha_{p}, 1^{n}\right\}$ is a partition of $N$, and $\alpha^{\prime}=\left\{\alpha_{1}, \alpha_{2}, \ldots, \alpha_{p}\right\}$ then

$$
\bar{d}_{X_{a}}(C) \leq\left(\begin{array}{c}
N \\
n
\end{array}\right)^{-1} \sum_{\varphi \in Q_{N-n, N}} \bar{d}_{X_{\alpha^{\prime}}}(C[\varphi \mid \varphi]) \operatorname{det}(C(\varphi \mid \varphi))
$$

with equality if $C$ is diagonal or both sides reduce to 0 .

Lemma 5. Suppose $A \in T_{n}, B \in T_{p}$, and $\gamma \in S_{n+p}$ satisfies:

(a) $\gamma^{2}=e$.

(b) If $1 \leq i \leq n$ and $\gamma(i) \neq i$ then $\gamma(i)>n$.

(c) If $n+1 \leq i \leq n+p$ and $\gamma(i) \neq i$ then $\gamma(i) \leq n$.

Then $\langle\gamma(A \otimes B), A \otimes B\rangle \geq 0$.

Proof. Conditions (a), (b) and (c) imply that $\gamma$ is a product of disjoint transpositions $(r s)$ such that $1 \leq r \leq n$ and $n+1 \leq s \leq n+p$. We claim that we may assume $\gamma=(1, n+1)(2, n+2) \cdots(k, n+k)$ where $k$ is the number of transpositions involved in $\gamma$, for if this is not the case then there exists $\alpha \in G(\{1,2, \ldots, n\})$ and $\beta=G(\{n+1, n+2, \ldots, n+p\})$ such that $\gamma=(\alpha \beta)^{-1}(1, n+1)(2, n+2) \cdots(k, n+k)(\alpha \beta) ;$ then

$$
\langle\gamma(A \otimes B), A \otimes B\rangle=\left\langle\gamma^{\prime}\left(\alpha^{\wedge} A \otimes \beta^{\vee} B\right), \alpha^{\wedge} A \otimes \beta^{\vee} B\right\rangle
$$

where $\gamma^{\prime}$ denotes $(1, n+1)(2, n+2) \cdots(k, n+k)$. Now, assuming $\gamma$ is $(1, n+1)(2, n+2) \cdots(k, n+k)$, we have $\langle\gamma(A \otimes B), A \otimes B\rangle$

$$
\begin{aligned}
= & \sum_{q_{1}=1}^{m} \ldots \sum_{q_{n+p}=1}^{m} \gamma(A \otimes B)\left(e_{q_{1}}, e_{q_{2}}, \ldots e_{q_{n+p}}\right) \overline{(A \otimes B)\left(e_{q_{1}}, e_{q_{2}}, \ldots, e_{q_{n+p}}\right)} \\
= & \sum_{q} A\left(e_{q_{n+1}}, \ldots, e_{q_{n+k}}, e_{q_{k+1}}, \ldots, e_{q_{n}}\right) B\left(e_{q_{1}}, \ldots, e_{q_{k}}, e_{q_{n+k+1}}, \ldots e_{q_{n+p}}\right) \\
& \times \overline{A\left(e_{q_{1}}, \ldots, e_{q_{k}}, e_{q_{k+1}}, \ldots, e_{q_{n}}\right)} \\
& \times \overline{B\left(e_{q_{n+1}}, \ldots, e_{q_{n+k}}, e_{q_{n+k+1}}, e_{q_{n+k+1}}, \ldots, e_{q_{n+p}}\right)}
\end{aligned}
$$

which, if for each $s, t, \Gamma_{s, t}$ denotes the set of all sequences of length $s$ each of whose terms is a member of $\{1,2, \ldots, t\}$, is the same as

$$
\sum_{q} \sum_{r} \sum_{s} \sum_{t} A\left(e_{s}, e_{r}\right) B\left(e_{q}, e_{t}\right) \overline{A\left(e_{q}, e_{r}\right) B\left(e_{s}, e_{t}\right)}
$$


where the summations are over $\Gamma_{k, m}, \Gamma_{n-k, m}, \Gamma_{k, m}$ and $\Gamma_{p-k, m}$ respectively, and $A\left(e_{s}, e_{r}\right)$ denotes $A\left(e_{s_{1}}, e_{s_{2}}, \ldots, e_{s_{k}}, e_{r_{1}}, e_{r_{2}}, \ldots, e_{r_{n-k}}\right)$, etc. The last expression above is the same as

$$
\begin{gathered}
\sum_{r, t}\left(\sum_{s} A\left(e_{s}, e_{r}\right) \overline{B\left(e_{s}, e_{t}\right)}\right)\left(\sum_{q} \overline{A\left(e_{q}, e_{r}\right)} B\left(e_{q}, e_{t}\right)\right) \\
=\sum_{r, t}\left|\sum_{s} A\left(e_{s}, e_{r}\right) B\left(e_{s}, e_{t}\right)\right|^{2} \geq 0 .
\end{gathered}
$$

Lemma 6. Let $\Delta=\left\{\delta_{1}, \delta_{2}, \ldots, \delta_{n+p}\right\} \subset\{1,2, \ldots, N+P\}, \Delta^{l}=\Delta \cap\{1,2$, $\ldots, N\}, \Delta^{r}=\Delta \cap\{N+1, N+2, \ldots, N+P\}$, and $k=\min \{n, p\}$. Suppose $n=\left|\Delta^{l}\right|$ and $p=\left|\Delta^{r}\right|$. Then

$$
\mathscr{S}(\Delta)=\left(\begin{array}{c}
n+p \\
n
\end{array}\right)^{-1} \sum_{i=0}^{k}\left(\begin{array}{c}
n \\
i
\end{array}\right)\left(\begin{array}{c}
p \\
i
\end{array}\right) \mathscr{S}\left(\Delta^{l}\right) \mathscr{S}\left(\Delta^{r}\right) \gamma_{i} \mathscr{S}\left(\Delta^{r}\right) \mathscr{S}\left(\Delta^{l}\right)
$$

where $\gamma_{i}=\prod_{s=1}^{i}\left(\delta_{s}, \delta_{n+s}\right), 1 \leq i \leq k$, and $\gamma_{0}=e$.

Proof. Let $H$ denote $G\left(\Delta^{l}\right) \cdot G\left(\Delta^{r}\right)$. It can be shown, see [2], that $\left\{\gamma_{0}, \gamma_{1}, \ldots\right.$, $\left.\gamma_{k}\right\}$ is a system of distinct representatives of the double cosets of $H$ in $G(\Delta)$. Moreover, if a list is constructed of all products of the form $\sigma \gamma_{i} \tau, \sigma, \tau \in H$, then each member of $H \gamma_{i} H$ will appear in the list $[(n-i) !(p-i) !(i !)]^{2}$ times. Letting $c_{i}$ denote the reciprocal of $\left[(n-i) !(p-i) !(i !)^{2}\right]$ we have:

$$
\begin{aligned}
(n+p) ! \mathscr{S}(\Delta) & =\sum_{\sigma \in G(\Delta)} \sigma=\sum_{i=0}^{k} c_{i} \sum_{\sigma, \tau \in H} \sigma \gamma_{i} \tau=\sum_{i=0}^{k} c_{i}\left(\sum_{\sigma \in H} \sigma\right) \gamma_{i}\left(\sum_{\tau \in H} \tau\right) \\
& =|H|^{2} \sum_{i=0}^{k} c_{i} \mathscr{S}\left(\Delta_{i}^{l}\right) \mathscr{S}\left(\Delta^{r}\right) \gamma_{i} \mathscr{S}\left(\Delta^{r}\right) \mathscr{S}\left(\Delta^{l}\right) \\
& =n ! p ! \sum_{i=0}^{k}\left(\begin{array}{c}
n \\
i
\end{array}\right)\left(\begin{array}{c}
p \\
i
\end{array}\right) \mathscr{S}\left(\Delta^{l}\right) \mathscr{S}\left(\Delta^{r}\right) \gamma_{i} \mathscr{S}\left(\Delta^{r}\right) \mathscr{S}\left(\Delta^{l}\right) .
\end{aligned}
$$

Therefore,

$$
\mathscr{S}(\Delta)=\left(\begin{array}{c}
n+p \\
n
\end{array}\right)^{-1} \sum_{i=0}^{k}\left(\begin{array}{c}
n \\
i
\end{array}\right)\left(\begin{array}{c}
p \\
i
\end{array}\right) \mathscr{S}\left(\Delta^{l}\right) \mathscr{S}\left(\Delta^{r}\right) \gamma_{i} \mathscr{S}\left(\Delta^{r}\right) \mathscr{S}\left(\Delta^{l}\right) .
$$

Theorem 5. Suppose $\Delta_{1}, \Delta_{2}, \ldots, \Delta_{k}$ are pairwise disjoint subsets of $\{1,2$, $\ldots, N+P\}$. For $1 \leq i \leq k$ let $\Delta_{i}^{l}=\Delta_{i} \cap\{1,2, \ldots, N\}$ and $\Delta_{i}^{r}=\Delta_{i} \cap\{N+1$, $N+2, \ldots, N+P\}$. Denote $\left|\Delta_{i}^{l}\right|$ by $\cdot n_{i},\left|\Delta_{i}^{r}\right|$ by $p_{i}$, and $n_{i}+p_{i}$ by $m_{i}$. Then

$$
\prod_{i=1}^{k}\left(\begin{array}{c}
m_{i} \\
n_{i}
\end{array}\right)\left\|\prod_{i=1}^{k} \mathscr{S}\left(\Delta_{i}\right)(A \otimes B)\right\|^{2} \geq\left\|\left(\prod_{i=1}^{k} \mathscr{S}\left(\Delta_{i}^{l}\right)\right)^{\wedge} A\right\|^{2}\left\|\left(\prod_{i=1}^{k} \mathscr{S}\left(\Delta_{i}^{r}\right)\right)^{\vee} B\right\|^{2}
$$


for each $A \in T_{N}$ and $B \in T_{P}$. Equivalently,

$$
\begin{aligned}
& \prod_{i=1}^{k}\left(\begin{array}{c}
m_{i} \\
n_{i}
\end{array}\right)\left\langle\prod_{i=1}^{k} \mathscr{S}\left(\Delta_{i}\right)(A \otimes B), A \otimes B\right\rangle \\
& \quad \geq\left\langle\left(\prod_{i=1}^{k} \mathscr{S}\left(\Delta_{i}^{l}\right)\right)^{\wedge} A, A\right\rangle\left\langle\left(\prod_{i=1}^{k} \mathscr{S}\left(\Delta_{i}^{r}\right)\right)^{\vee} B, B\right\rangle .
\end{aligned}
$$

Proof. Let $\Delta_{i}=\left\{\delta_{i 1}, \delta_{i 2}, \ldots, \delta_{i m_{i}}\right\}$ where we assume $\delta_{i 1}<\delta_{i 2}<\cdots<$ $\delta_{i m_{i}}, 1 \leq i \leq k$. For $1 \leq j \leq c_{i}, c_{i}=\min \left\{n_{i}, p_{i}\right\}$, we let $\gamma_{i j}$ denote $\prod_{i=1}^{j}\left(\delta_{i s}, \delta_{i, s+n_{i}}\right)$, a product of disjoint transpositions. Now, by Lemma 6 ,

$$
\begin{aligned}
\prod_{i=1}^{k}\left(\begin{array}{c}
m_{i} \\
n_{i}
\end{array}\right) \prod_{i=1}^{k} \mathscr{S}\left(\Delta_{i}\right) \\
\quad=\prod_{i=1}^{k}\left\{\sum_{j=0}^{c_{i}}\left(\begin{array}{c}
n_{i} \\
j
\end{array}\right)\left(\begin{array}{c}
p_{i} \\
j
\end{array}\right) \mathscr{S}\left(\Delta_{i}^{l}\right) \mathscr{S}\left(\Delta_{i}^{r}\right) \gamma_{i j} \mathscr{S}\left(\Delta_{i}^{r}\right) \mathscr{S}\left(\Delta_{i}^{l}\right)\right\} \\
\quad=\sum_{j_{1}=0}^{c_{1}} \sum_{j_{2}=0}^{c_{2}} \ldots \sum_{j_{k}=0}^{c_{k}} \prod_{i=1}^{k}\left(\begin{array}{c}
n_{i} \\
j_{i}
\end{array}\right)\left(\begin{array}{c}
p_{i} \\
j_{i}
\end{array}\right) \mathscr{S}\left(\Delta_{i}^{l}\right) \mathscr{S}\left(\Delta_{i}^{r}\right) \gamma_{i j_{i}} \mathscr{S}\left(\Delta_{i}^{r}\right) \mathscr{S}\left(\Delta_{i}^{l}\right) .
\end{aligned}
$$

Given $A \in T_{N}$ and $B \in T_{P}$ we have

$$
\begin{aligned}
& \left\langle\left(\prod_{i=1}^{k} \mathscr{S}\left(\Delta_{i}^{l}\right) \mathscr{S}\left(\Delta_{i}^{r}\right) \gamma_{i j_{i}} \mathscr{S}\left(\Delta_{i}^{r}\right) \mathscr{S}\left(\Delta_{i}^{l}\right)\right)(A \otimes B), A \otimes B\right\rangle \\
& =\left\langle\left(\prod_{i=1}^{k} \gamma_{i j_{i}}\right)\left(\left(\prod_{i=1}^{k} \mathscr{S}\left(\Delta_{i}^{l}\right)\right)^{\wedge} A \otimes\left(\prod_{i=1}^{k} \mathscr{S}\left(\Delta_{i}^{r}\right)\right)^{\vee} B\right),\right. \\
& =\left\langle\left(\prod_{i=1}^{k} \gamma_{i j_{i}}\right)\left(A^{\prime} \otimes B^{\prime}\right), A^{\prime} \otimes B^{\prime}\right\rangle
\end{aligned}
$$

where $A^{\prime}=\left(\prod_{i=1}^{k} \mathscr{S}\left(\Delta_{i}^{l}\right)\right)^{\wedge} A$ and $B^{\prime}=\left(\prod_{i=1}^{k} \mathscr{S}\left(\Delta_{i}^{r}\right)\right)^{\vee} B$. But $\prod_{i=1}^{k} \gamma_{i j_{i}}$ is a product of disjoint transpositions ( $r s$ ) such that $1 \leq r \leq N$ and $N+1 \leq s \leq$ $N+P$. Therefore,

$$
\left\langle\left(\prod_{i=1}^{k} \gamma_{i j_{l}}\right)\left(A^{\prime} \otimes B^{\prime}\right), A^{\prime} \otimes B^{\prime}\right\rangle \geq 0
$$


by Lemma 5 . Therefore, by setting each $j_{i}=0$ we obtain the inequality

$$
\begin{aligned}
\prod_{i=1}^{k}\left(\begin{array}{c}
m_{i} \\
n_{i}
\end{array}\right)\left\langle\prod_{i=1}^{k} \mathscr{S}\left(\Delta_{i}\right)(A \otimes B), A \otimes B\right\rangle \\
\quad \geq\left\langle\prod_{i=1}^{k} \mathscr{S}\left(\Delta_{i}^{l}\right) \mathscr{S}\left(\Delta_{i}^{r}\right) \mathscr{S}\left(\Delta_{i}^{r}\right) \mathscr{S}\left(\Delta_{i}^{l}\right)(A \otimes B), A \otimes B\right\rangle \\
\quad=\left\langle\left(\prod_{i=1}^{k} \mathscr{S}\left(\Delta_{i}^{l}\right)\right)^{\wedge} A, A\right\rangle\left\langle\left(\prod_{i=1}^{k} \mathscr{S}\left(\Delta_{i}^{r}\right)\right)^{\vee} B, B\right\rangle .
\end{aligned}
$$

Suppose $\alpha=\left\{\alpha_{1}, \alpha_{2}, \ldots, \alpha_{s}\right\}$ is a partition of $N$ and imagine the associated node diagram. If the diagram is cut into two pieces along a vertical line not containing any nodes then we obtain two new node diagrams each associated with a partition which in turn is associated with a character. We denote the new partitions by $\alpha_{l}$, the partition associated with the node diagram on the left, and $\alpha_{r}$, the partition associated with the node diagram on the right. We may now induce the tensor product of $\widehat{X}_{\alpha_{l}}$ and $\widehat{X}_{\alpha_{r}}$ to $S_{N}$, thus obtaining $\left(\widehat{X}_{\alpha_{l}} \otimes \widehat{X}_{\alpha_{r}}\right)^{\uparrow}$, and investigate the possibility that there exists inequality between $\widehat{X}_{\alpha}$ and $\left(\widehat{\widehat{X}}_{\alpha_{l}} \otimes \widehat{X}_{\alpha_{r}}\right)^{\uparrow}$. Theorem 6 guarantees that

$$
\widehat{X}_{\alpha} \geq\left(\widehat{\widehat{X}}_{\alpha_{l} \otimes \widehat{X}_{\alpha_{r}}}\right)^{\uparrow} \text {. }
$$

Theorem 6. Suppose $\alpha=\left\{\alpha_{1}, \alpha_{2}, \ldots \alpha_{s}\right\}$ is a partition of $N$. Let $\rho$ and $t$ be positive integers such that $t \leq s, \alpha_{i}>\rho$ for $1 \leq i \leq t$, and $\rho \geq \alpha_{j}$ for $t<j \leq s$. Let

$$
\alpha_{l}=\left\{\rho^{t}, \alpha_{t+1}, \alpha_{t+2}, \ldots, \alpha_{s}\right\} \text { and } \alpha_{r}=\left\{\alpha_{1}-\rho, \alpha_{2}-\rho, \ldots, \alpha_{t}-\rho\right\} .
$$

Then, $\left\langle\widehat{X}_{\alpha} Y, Y\right\rangle \geq\left\langle\left(\widehat{\widehat{X}}_{\alpha_{l}} \otimes \widehat{X}_{\alpha_{r}}\right)^{\uparrow} Y, Y\right\rangle$ for each decomposable $Y \in T_{N}$.

Proof. We create a Young diagram $D_{\alpha}$ by filling the first column of the $\alpha$ frame with $1,2, \ldots, s$, the second column with $s+1, s+2, \ldots$ etc. We consider this tableaux to be the adjunction of two tableaux, one associated with $\alpha_{l}$ and containing the integers $1,2, \ldots, N-n$, where $n=\sum_{i=1}^{t} \alpha_{i}-t \rho$, the other associated with $\alpha_{r}$ and containing $N-n+1, N-n+2, \ldots, N$.

We let $\Delta_{1}, \Delta_{2}, \ldots, \Delta_{s}$ be the row sets of $D_{\alpha}$ and, for $1 \leq i \leq s, \Delta_{i}^{l}=$ $\Delta_{i} \cap\{1,2, \ldots, N-n\}$ and $\Delta_{i}^{r}=\Delta_{i} \cap\{N-n+1, N-n+2, \ldots, N\}$. Denoting $r_{\alpha}$ by $r$ and $c_{\alpha}$ by $c$ we observe that

$$
r=\prod_{i=1}^{s} \alpha_{i} ! \mathscr{S}\left(\Delta_{i}\right)
$$

that

$$
r_{\alpha_{l}}=\left[(\rho !)^{t} \prod_{i=1}^{t} \mathscr{S}\left(\Delta_{i}^{l}\right) \prod_{i=t+1}^{s} \alpha_{i} ! \mathscr{S}\left(\Delta_{i}\right)\right]^{\wedge}
$$


and

$$
r_{\alpha_{r}}=\left[\prod_{i=1}^{t}\left(\alpha_{i}-\rho\right) ! \mathscr{S}\left(\Delta_{i}^{r}\right)\right]^{\vee},
$$

where the tableaux associated with $\alpha_{l}$ is obtained from $D_{\alpha}$ by deleting the last $\alpha_{1}-\rho$, columns, and the tableaux associated with $\alpha_{r}$ is obtained from $D_{\alpha}$ by deleting the first $\rho$ columns and then subtracting $N-n$ from each entry in the resulting tableaux. that

Letting $c^{\prime}$ denote $c_{\alpha_{l}}$ and $c^{\prime \prime}$ denote $c_{\alpha_{r}}$ we observe that $c=c^{\prime} \otimes c^{\prime \prime}$ and

$$
\begin{aligned}
\widehat{X}_{\alpha} & =(N !)^{-1} \sum_{\sigma} \sigma r c \sigma^{-1}=\left(N !\left|C_{\alpha}\right|\right)^{-1} \sum_{\sigma} \sigma c r c \sigma^{-1} \\
& =\left(N !\left|C_{\alpha}\right|\right)^{-1} \sum_{\sigma} \sigma\left(c^{\prime} \otimes c^{\prime \prime}\right) r\left(c^{\prime} \otimes c^{\prime \prime}\right) \sigma^{-1}
\end{aligned}
$$

Therefore, for decomposable $Y \in T_{N}$ we have

$$
\begin{aligned}
\left\langle\widehat{X}_{\alpha} Y, Y\right\rangle & =K \sum_{\sigma \in S_{N}}\left\langle\sigma\left(c^{\prime} \otimes c^{\prime \prime}\right) r\left(c^{\prime} \otimes c^{\prime \prime}\right) \sigma^{-1} Y, Y\right\rangle \\
& =K \sum_{\sigma}\left\langle r\left(c^{\prime} \otimes c^{\prime}\right)\left(Y_{1}(\sigma) \otimes Y_{2}(\sigma)\right),\left(c^{\prime} \otimes c^{\prime \prime}\right)\left(Y_{1}(\sigma) \otimes Y_{2}(\sigma)\right)\right\rangle \\
& =K \prod_{i=1}^{s} \alpha_{i} ! \sum_{\sigma}\left\langle\prod_{i=1}^{s} \mathscr{S}\left(\Delta_{i}\right)\left(c^{\prime} Y_{1}(\sigma) \otimes c^{\prime \prime} Y_{2}(\sigma)\right), c^{\prime} Y_{1}(\sigma) \otimes c^{\prime \prime} Y_{2}(\sigma)\right\rangle \\
& =K \prod_{i=1}^{s} \alpha_{i} ! \sum_{\sigma}\left\langle\prod_{i=1}^{t} \mathscr{S}\left(\Delta_{i}\right)\left(A_{\sigma} \otimes B_{\sigma}\right), A_{\sigma} \otimes B_{\sigma}\right\rangle
\end{aligned}
$$

where $K=\left(N !\left|C_{\alpha}\right|\right)^{-1}, Y_{1}(\sigma)$ and $Y_{2}(\sigma)$ are as in Theorem 3,

$$
A_{\sigma}=\left(\prod_{i=t+1}^{s} \mathscr{S}\left(\Delta_{i} u\right)\right)^{\wedge} c^{\prime} Y_{1}(\sigma), \quad \text { and } \quad B_{\sigma}=c^{\prime \prime} Y_{2}(\sigma)
$$

Now, applying Theorem 5, we have

$$
\begin{aligned}
\left\langle\widehat{X}_{\alpha} Y, Y\right\rangle \geq & K \prod_{i=1}^{s} \alpha_{i} ! \prod_{j=1}^{t}\left(\begin{array}{c}
\alpha_{j} \\
\rho
\end{array}\right)^{-1}\left\langle\left(\prod_{i=1}^{t} \mathscr{S}\left(\Delta_{i}^{l}\right)\right)^{\wedge} A_{\sigma}, A_{\sigma}\right\rangle \\
& \times\left\langle\left(\prod_{i=1}^{t} \mathscr{S}\left(\Delta_{i}^{r}\right)\right)^{\vee} B_{\sigma}, B_{\sigma}\right\rangle \\
= & K(\rho !)^{t} \prod_{i=1}^{t}\left(\alpha_{i}-\rho\right) ! \prod_{j=t+1}^{s} \alpha_{j} ! \sum_{\sigma}\left\langle\psi_{1} \psi_{2} c^{\prime} Y_{1}(\sigma), \psi_{2} c^{\prime} Y_{1}(\sigma)\right\rangle \\
& \times\left\langle\psi_{3} c^{\prime \prime} Y_{2}(\sigma), c^{\prime \prime} Y_{2}(\sigma)\right\rangle
\end{aligned}
$$

where $\psi_{1}=\left(\prod_{i=1}^{t} \mathscr{S}\left(\Delta_{i}^{l}\right)\right)^{\wedge}, \psi_{2}=\left(\prod_{i=t+1}^{s} \mathscr{S}\left(\Delta_{i}\right)\right)^{\wedge}$, and $\psi_{3}=\left(\prod_{i=1}^{t} \mathscr{S}\left(\Delta_{i}^{r}\right)\right)^{\vee}$. Since $\psi_{1} \psi_{2}=\psi_{2} \psi_{1}, r_{\alpha_{l}}=(\rho !)^{t} \prod_{i=t+1}^{s} \alpha_{i} ! \psi_{1} \psi_{2}$, and $r_{\alpha_{r}}=\prod_{i=1}^{t}\left(\alpha_{i}-\rho\right) ! \psi_{3}$ the 
above is equal to

$$
K \sum_{\sigma}\left\langle c^{\prime} r_{\alpha_{l}} c^{\prime} Y_{1}(\sigma), Y_{1}(\sigma)\right\rangle\left\langle c^{\prime \prime} r_{\alpha_{r}} c^{\prime \prime} Y_{2}(\sigma), Y_{2}(\sigma)\right\rangle
$$

Letting $\mathscr{U}$ denote the set of all members of $S_{N}$ that increase on $\{1,2, \ldots$, $N-n\}$ and $\{N-n+1, N-n+2, \ldots, N\}$, and $H$ denote

$$
G(\{1,2, \ldots, N-n\}) \cdot G(\{N-n+1, N-n+2, \ldots N\}),
$$

and applying Lemmas 2, 3, and 4 as well as formulas (2.6), (2.7), and (2.10), we see that the last of the above expressions is

$$
\begin{aligned}
& =K \sum_{\varphi \in \mathscr{U}} \sum_{\tau \in H}\left\langle c^{\prime} r_{\alpha_{l}} c^{\prime}\left(\tau^{\wedge}\right)^{-1} Y_{1}(\varphi),\left(\tau^{\wedge}\right)^{-1}\right\rangle\left\langle c^{\prime \prime} r_{\alpha_{r}} c^{\prime \prime}\left(\tau^{\vee}\right)^{-1} Y_{2}(\varphi),\left(\tau^{\vee}\right)^{-1} Y_{2}(\varphi)\right\rangle \\
& =K \sum_{\varphi \in \mathscr{U}} \sum_{\mu \in S_{n}} \sum_{\theta \in S_{N-n}}\left\langle\mu c_{\alpha_{l}} r_{\alpha_{l}} c_{\alpha_{l}} \mu^{-1} Y_{1}(\varphi), Y_{1}(\varphi)\right\rangle\left\langle\theta c_{\alpha_{r}} r_{\alpha_{r}} c_{\alpha_{r}} \theta^{-1} Y_{2}(\varphi), Y_{2}(\varphi)\right\rangle \\
& =K\left|C_{\alpha_{l}} \| C_{\alpha_{r}}\right| \sum_{\varphi} \sum_{\mu, \theta}\left\langle\mu r_{\alpha_{l}} c_{\alpha_{l}} \mu^{-1} Y_{1}(\varphi), Y_{1}(\varphi)\right\rangle\left\langle\theta r_{\alpha_{r}} c_{\alpha_{r}} \theta^{-1} Y_{2}(\varphi), Y_{2}(\varphi)\right\rangle \\
& =\left(\begin{array}{c}
N \\
n
\end{array}\right)^{-1} \sum_{\varphi \in \mathscr{U}}\left\langle\widehat{X}_{\alpha_{l}} Y_{1}(\varphi), Y_{1}(\varphi)\right\rangle\left\langle\widehat{X}_{\alpha_{r}} Y_{2}(\varphi), Y_{2}(\varphi)\right\rangle \\
& =\left\langle\left(\widehat{\widehat{X}}_{\alpha_{l}} \otimes \widehat{X}_{\alpha_{r}}\right)^{\uparrow} Y, Y\right\rangle \text {. }
\end{aligned}
$$

For each partition $\alpha=\left\{\alpha_{1}, \alpha_{2}, \ldots, \alpha_{s}\right\}$ we shall associate a derived partition $\alpha^{\prime}$ which, if $t$ denotes the smallest positive integer such that $\alpha_{t}>\alpha_{t+1}$, is equal to $\left\{\alpha_{1}-1, \alpha_{2}-1, \ldots, \alpha_{t}-1, \alpha_{t+1}, \alpha_{t+2}, \ldots, \alpha_{s}, 1^{t}\right\}$. The following result, perhaps our most appealing in an aesthetic sense, follows immediately from Theorems 3 and 6.

Theorem 7. If $\alpha$ is a partition and $\alpha^{\prime}$ is its derived partition then $\widehat{X}_{\alpha} \geq \widehat{X}_{\alpha^{\prime}}$. Proof. Let $\alpha=\left\{\alpha_{1}, \alpha_{2}, \ldots, \alpha_{s}\right\}=\left\{(\rho+1)^{t}, \alpha_{t+1}, \alpha_{t+2}, \ldots, \alpha_{s}\right\}$ where $\rho \geq$ $\alpha_{t+1}$. Let $\alpha_{l}=\left\{\rho^{t}, \alpha_{t+1}, \alpha_{t+2}, \ldots, \alpha_{s}\right\}$ and $\alpha_{r}=\left\{1^{t}\right\}$. Letting $\mathscr{E}$ denote $\left(\widehat{\widehat{X}}_{\alpha_{l}} \otimes \widehat{X}_{\alpha_{r}}\right)^{\uparrow}$ we have $\widehat{X}_{\alpha} \geq \mathscr{E}$ by Theorem 6 , and $\mathscr{E} \geq \widehat{X}_{\alpha^{\prime}}$ by Theorem 3 . Hence, $\widehat{X}_{\alpha} \geq \widehat{X}_{\alpha^{\prime}}$ as required.

Successive application of Theorem 7 to a partition $\alpha$ yields the sequence

$$
\widehat{X}_{\alpha} \geq \widehat{X}_{\alpha^{\prime}} \geq \widehat{X}_{\alpha^{\prime \prime}} \geq \widehat{X}_{\alpha^{(3)}} \geq \cdots \geq \widehat{X}_{\alpha^{(k)}}=\varepsilon
$$

where $k=\alpha_{1}-1$ and $\varepsilon$ is the signum function. The corresponding sequence of matrix inequalities is, for $C \in \mathscr{H}_{N}$,

$$
\bar{d}_{X_{\prime^{\prime}}}(C) \geq \bar{d}_{X_{\alpha^{\prime}}}(C) \geq \bar{d}_{X_{\sigma^{\prime \prime}}}(C) \geq \cdots \geq \operatorname{det}(C)
$$

a dramatic improvement over Schur's result (1.3).

In a recent paper, see [10], Heyfron has shown that if $\alpha=\left\{q+1,1^{N-q-1}\right\}$ and $\beta=\left\{q, 1^{N-q}\right\}$ then $\bar{d}_{X_{n}}(C) \geq \bar{d}_{X_{\beta}}(C)$ for each $C \in \mathscr{H}_{N}$. Since $\beta=\alpha^{\prime}$ this result is merely a special case of Theorem 7 . 
But Theorems 4 and 6 applied separately give us a generalization of Heyfron's result as well as an improvement on the per-det inequalities for the singlehook immanents obtained by Merris and Watkins in [11]. Following [11] we let $\lambda_{k}$, for $k=1,2, \ldots, n$, denote the irreducible character associated with $\left\{k, 1^{n-k}\right\}$ and we abbreviate $d_{\lambda_{k}}$ with $d_{k}$. Then Merris and Watkins have shown that

$$
d_{k}(C) \leq \sum_{\varphi \in Q_{k, n}} \operatorname{per}(C[\varphi \mid \varphi]) \operatorname{det}(C(\varphi \mid \varphi))
$$

for each $C \in \mathscr{H}_{n}$ where $k \in\{1,2, \ldots, n\}$. Since the degree of $\lambda_{k}$ is $\left(\begin{array}{c}n-1 \\ k-1\end{array}\right)$, the above is equivalent to

$$
\bar{d}_{k}(C) \leq(n / k)\left(\begin{array}{l}
n \\
k
\end{array}\right)^{-1} \sum_{\varphi \in Q_{k, n}} \operatorname{per}(C[\varphi \mid \varphi]) \operatorname{det}(C(\varphi \mid \varphi))
$$

for each $C \in \mathscr{H}_{n}$ and $k \in\{1,2, \ldots, n\}$. But direct application of Theorem 4 gives

$$
\bar{d}_{k}(C) \leq\left(\begin{array}{l}
n \\
k
\end{array}\right)^{-1} \sum_{\varphi \in Q_{k, n}} \operatorname{per}(C[\varphi \mid \varphi]) \operatorname{det}(C(\varphi \mid \varphi)),
$$

hence

$$
d_{k}(C) \leq(k / n) \sum_{\varphi \in Q_{k, n}} \operatorname{per}(C[\varphi \mid \varphi]) \operatorname{det}(C(\varphi \mid \varphi))
$$

for each $c \in \mathscr{H}_{n}$ and $k \in\{1,2, \ldots, n\}$.

Applying Theorem 6 to $\lambda_{k+1}, k \in\{0,1, \ldots, n-1\}$, with $p=1, t=1$, and $s=n-k$ we have $\alpha_{l}=\left\{1^{n-k}\right\}$ and $\alpha_{r}=\{k\}$ so

$$
\bar{d}_{k+1}(C) \geq\left(\begin{array}{l}
n \\
k
\end{array}\right)^{-1} \sum_{\varphi \in Q_{n-k, n}} \operatorname{det}(C[\varphi \mid \varphi]) \operatorname{per}(C(\varphi \mid \varphi))
$$

hence

$$
\dot{\bar{d}}_{k+1}(C) \geq\left(\begin{array}{l}
n \\
k
\end{array}\right)^{-1} \sum_{\varphi \in Q_{k, n}} \operatorname{per}(C[\varphi \mid \varphi]) \operatorname{det}(C(\varphi \mid \varphi)) .
$$

Combining this with the above we have

$$
\bar{d}_{k}(C) \leq\left(\begin{array}{l}
n \\
k
\end{array}\right)^{-1} \sum_{\varphi \in Q_{k, n}} \operatorname{per}(C[\varphi \mid \varphi]) \operatorname{det}(C(\varphi \mid \varphi)) \leq \bar{d}_{k+1}(C)
$$

for each $C \in \mathscr{H}_{n}$ and $k \in\{1,2, \ldots, n-1\}$.

To obtain the James-Liebeck result, namely that if $\beta=\left\{p, q, 1^{r}\right\}, p \geq q$ and $p+q+r=N$, then

$$
\operatorname{per}(C) \geq \bar{d}_{X_{\beta}}(C)
$$

for each $C \in \mathscr{H}_{N}$, we set $\alpha=\{p+r, q\}$ and note that $\alpha^{(i)}=\left\{p+r-i, q, 1^{i}\right\}$, $1 \leq i \leq r$. Hence, $\alpha^{(r)}=\beta$, and

$$
\bar{d}_{X_{u^{\prime}}}(C) \geq \bar{d}_{X_{n^{\prime}}}(C) \geq \bar{d}_{X_{n^{\prime \prime}}}(C) \geq \cdots \geq \bar{d}_{X_{n^{\prime \prime}}}(C)
$$


for each $C \in \mathscr{H}_{N}$. But, the author has shown in [9], that $\bar{d}_{X_{\alpha}}(C) \leq \operatorname{per}(C)$ for any 2-term partition $\alpha$. We thus have the following strengthened version of the James-Liebeck result:

$$
\operatorname{per}(C) \geq \bar{d}_{X_{\alpha}}(C) \geq \bar{d}_{X_{\alpha^{\prime}}}(C) \geq \cdots \geq \bar{d}_{X_{\alpha^{(r)}}}(C)=\bar{d}_{X_{\beta}}(C)
$$

for $C \in \mathscr{H}_{N}$. But Theorem 7 gives even more since $\bar{d}_{X_{\alpha^{(i)}}}(C) \geq \bar{d}_{X_{\alpha^{(i+1)}}}(C)$ for $1 \leq i \leq p+r-2$.

The partition $\alpha^{\prime}$ is obtained from $\alpha$ in the simplest manner by referring to the corresponding node diagram. For example, if $\alpha=\left\{5^{2}, 4,2^{2}\right\}$ then the node diagram is

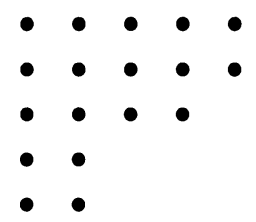

To obtain the node diagram for $\alpha^{\prime}$ simply remove the last column of dots from the above diagram and append it to the first column, thus obtaining

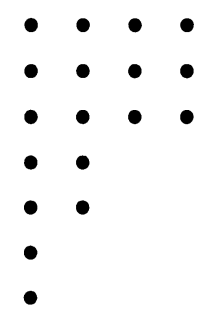

whose corresponding partition is $\left\{4^{3}, 2^{2}, 1^{2}\right\}$. Continuing in this manner we obtain $\alpha^{\prime \prime}=\left\{3^{3}, 2^{2}, 1^{5}\right\}, \alpha^{(3)}=\left\{2^{5}, 1^{8}\right\}$, and $\alpha^{(4)}=\left\{1^{18}\right\}$.

\section{REFERENCES}

1. J. W. Neuberger, Norm of symmetric product compared with norm of tensor product, Linear and Multilinear Algebra 2 (1974), 115-122.

2. T. H. Pate, A continuous analogue of the Lieb-Neuberger inequality, Houston J. Math. 12 (1986), 225-234.

3. I. Shur, Uber endliche Gruppen and Hermitische Formen, Math. Z. 1 (1918), 184-207.

4. M. Marcus, On two classical results of I. Schur, Bull. Amer. Math. Soc. 70 (1964), 685-688.

5. __ The Hadamard theorem for permanents, Proc. Amer. Math. Soc. 15 (1964), 967-975.

6. E. H. Lieb, Proofs of some conjectures on permanents, J. Math. Mech. 16 (1966), 127-134.

7. M. Marcus, Finite dimensional multilinear algebra, Part II, Dekker, New York, 1975.

8. G. D. James and M. Liebeck, Permanents and immanents of Hermitian matrices, Proc. London Math. Soc. (3) 55 (1987), 243-265.

9. T. H. Pate, Permanental dominance and the Soules conjecture for certain right ideals in the group algebra, Linear and Multilinear Algebra (to appear).

10. Peter Heyfron, Immanent dominance orderings for hook partitions, Linear and Multilinear Algebra 24 (1988), 65-78. 
11. R. Merris and W. Watkins, Inequalities and identities for generalized matrix functions, Linear Algebra Appl. 64 (1985), 223-242.

12. M. A. Naimark and A. I. Stern, Theory of group representations, Grundlehren Math. Wiss. 246 (1982).

13. J. P. Serre, Linear representations of finite groups, Graduate Texts in Math., Springer-Verlag, New York, Heidelberg, and Berlin, 1987.

14. T. H. Pate, Generalizing the Fischer inequality, Linear Algebra Appl. 92 (1987), 1-15.

Department of Mathematics, 100 Mathematics Building, 231 West 18th Avenue, Columbus State University, Columbus, Ohio 43210

Current address: Department of Mathematics, Auburn University, Auburn, Alabama, 36849 